ORIGINAL ARTICLE

\title{
Susceptibilities of Neisseria gonorrhoeae to fluoroquinolones and other antimicrobial agents in Hyogo and Osaka, Japan
}

\author{
K Shigemura, H Okada, T Shirakawa, K Tanaka, S Arakawa, S Kinoshita, A Gotoh, S Kamidono
}

Sex Transm Infect 2004;80:105-107. doi: 10.1136/sti.2003.006908

See end of article for authors' affiliations

......................

Correspondence to: Hiroshi Okada MD, PhD, Department of Urology, Tokyo University School of Medicine, 2-11-1 Kaga, Itabashi-Ku, Tokyo 1738605, Japan; okada@ med.teikyo-u.ac.jp

Accepted for publication 21 October 2003

\begin{abstract}
Objectives: Decreasing susceptibility of Neisseria gonorrhoeae to fluoroquinolones has been reported in several countries. Knowledge of local $N$ gonorrhoeae susceptibilities to various antimicrobials is important for establishing a rational treatment strategy in each region.

Methods: Isolates of $N$ gonorrhoeae from male urethritis patients attending four urological clinics in Hyogo and Osaka prefectures in Japan were collected during 2002. The MICs for nine antimicrobials: penicillin $G$, tetracycline, cefixime, ceftriaxone, levofloxacin, gatifloxacin, ciprofloxacin, moxifloxacin, and spectinomycin were determined for each isolate. All isolates were also tested for $\beta$ lactamase producing profiles.

Results: Among the 87 isolates obtained, only one isolate was revealed to produce $\beta$ lactamase. MIC 90 values for ciprofloxacin, levofloxacin, gatifloxacin, and moxifloxacin were over $8 \mu \mathrm{g} / \mathrm{ml}$, over $8 \mu \mathrm{g} / \mathrm{ml}$, $4 \mu \mathrm{g} / \mathrm{ml}$, and $2 \mu \mathrm{g} / \mathrm{ml}$, respectively. The proportion of isolates resistant to fluoroquinolones was over $60 \%$ (ciprofloxacin, 70.1\%; levofloxacin, 65.5\%; gatifloxacin, 70.1\%). Chromosomally mediated penicillin and tetracycline resistance was identified in $12.6 \%$ and $33.3 \%$ of the isolates. MIC90 values for cefixime and ceftriaxone and were $0.5 \mu \mathrm{g} / \mathrm{ml}$ and $0.0063 \mu \mathrm{g} / \mathrm{ml}$. All isolates were sensitive to ceftriaxone and $90.8 \%$ of them were sensitive to cefixime. MIC 90 for spectinomycin was $32 \mu \mathrm{g} / \mathrm{ml}$ and all isolates were sensitive to it. Fluoroquinolone resistance correlated significantly with MICs for penicillin $G$ but not tetracycline. Conclusion: Ceftriaxone and spectinomycin demonstrated lower MICs and so are recommended for $\mathrm{N}$ gonorrhoeae. Susceptibilities of $N$ gonorrhoeae should be monitored periodically by region.
\end{abstract}

$\mathrm{N}$ eisseria gonorrhoeae remains one of the most common sexually transmitted pathogens in developing and developed countries. Over the past decade, strains of $N$ gonorrhoeae have been reported to develop high levels of resistance against several antimicrobial agents previously used for treatment of gonorrhoea. ${ }^{12}$

Until several years ago, fluoroquinolone regimens had been recommended for the treatment of gonorrhoea; however, the emergence of gonococcal isolates with reduced susceptibility or resistance to fluoroquinolones has been a significant concern in several countries, including Japan. The recommended therapy has been altered accordingly.

Trends concerning resistance have varied from country to country and also between areas in a given country. We investigated MICs of $N$ gonorrhoeae isolated from men with urethritis in an urban area of Japan to establish a rational treatment strategy appropriate to the area.

\section{METHODS}

\section{Clinical specimens}

Urine or urethral swab specimens were obtained from male patients with urethritis treated at four urological clinics in Hyogo and Osaka prefectures in Japan in 2002. Posttreatment isolates and other repeat isolates from the same patients were excluded from the study. All specimens were transferred to the clinical laboratory at our institution within 2 hours of collection.

\section{Antimicrobial susceptibility testing}

MICs for all isolates were determined using the agar plate dilution method, with a GC agar base (Becton Dickinson, Cockeysville, MD, USA) containing 1\% Iso VitaleX (Becton Dickinson) and serial twofold dilutions of antimicrobial agents. MICs at which $50 \%$ and $90 \%$ of the isolates tested were inhibited were defined as the $\mathrm{MIC}_{50}$ and $\mathrm{MIC}_{90}$. We used $N$ gonorrhoeae ATCC 49226 as a quality control in susceptibility testing. $\beta$ lactamase production was assayed using nitrocefin discs (BBL Cefinase; Becton Dickinson) with Staphylococcus aureus ATCC 25923 as a negative control.

The antimicrobial agents tested were penicillin G, tetracycline, cefixime, ceftriaxone, levofloxacin, gatifloxacin, ciprofloxacin, moxifloxacin, and spectinomycin.

Antimicrobial susceptibilities for strains were defined according to the MIC ranges listed in the National Committee for Clinical Laboratory Standards (NCCLS) 2002 guidelines (table 1). ${ }^{4}$ The isolates were sequentially grouped into mutually exclusive categories according to the NCCLS guidelines as follows: penicillinase producing $N$ gonorrhoeae (PPNG); plasmid mediated tetracycline resistant $N$ gonorrhoeae (TRNG); chromosomally mediated penicillin resistant $N$ gonorrhoeae (CMPR); chromosomally mediated tetracycline resistant $N$ gonorrhoeae (CMTR); and chromosomally mediated penicillin and tetracycline resistant $N$ gonorrhoeae (CMRNG) according to the paper by Tanaka et al. ${ }^{3}$

\section{Statistical analysis}

Median MIC values were compared by the Mann-Whitney U test using a software package (StatView; Abacus Concepts, Berkeley, CA, USA). Statistical significance was set at 0.05.

\section{RESULTS}

Susceptibilities for fluoroquinolones

$N$ gonorrhoeae was isolated from urine or urethral swab specimens of 87 male patients with urethritis; 67 strains (77\%) were obtained in Hyogo prefecture, and 20 (23\%) in 
Table 1 Antimicrobial susceptibility and prevalence of $N$ gonorrhoeae susceptible to each drug

\begin{tabular}{|c|c|c|c|c|c|c|}
\hline \multirow[b]{2}{*}{ Antimicrobial agents } & \multirow{2}{*}{$\begin{array}{l}\mathrm{MIC}_{50} \\
(\mu \mathrm{g} / \mathrm{ml})\end{array}$} & \multirow{2}{*}{$\begin{array}{l}\text { MIC }_{90} \\
(\mu \mathrm{g} / \mathrm{ml})\end{array}$} & \multirow{2}{*}{$\begin{array}{l}\text { MIC range } \\
(\mu \mathrm{g} / \mathrm{ml})\end{array}$} & \multicolumn{3}{|c|}{ MIC range ( $\mu \mathrm{g} / \mathrm{ml})$ of interpretive standard (No of isolates) } \\
\hline & & & & Sensitive* & Intermediate* & Resistant* \\
\hline Penicillin G & 0.5 & 2 & $<0.031-64$ & $\leqslant 0.06(9 / 87)$ & $0.12-1(61 / 87)$ & $\geqslant 2(17 / 87)$ \\
\hline Tetracycline & 1 & 8 & $<0.031-64$ & $\leqslant 0.25(16 / 87)$ & $0.5-1(34-87)$ & $\geqslant 2(17 / 87)$ \\
\hline Cefixime & 0.125 & 0.5 & $<0.004-0.5$ & $\leqslant 0.25(79 / 87)$ & & $\geqslant 0.5(8 / 87)$ \\
\hline Ceftriaxone & 0.016 & 0.063 & $<0.004-0.063$ & $\leqslant 0.25(87 / 87)$ & & $\geqslant 0.5(0 / 87)$ \\
\hline Ciprofloxacin & 4 & $>8$ & $<0.002->8$ & $\leqslant 0.06(9 / 87)$ & $0.12-0.5(18 / 87)$ & $\geqslant 1(60 / 87)$ \\
\hline Levofloxacin & 4 & $>8$ & $<0.002->8$ & $\leqslant 0.25(24 / 87)$ & $0.15-1(5 / 87)$ & $\geqslant 2(58 / 87)$ \\
\hline Gatifloxacin & 1 & 4 & $<0.002->8$ & $\leqslant 0.125(19 / 87)$ & $0.25(7 / 87)$ & $\geqslant 0.5(61 / 87)$ \\
\hline Spectinomycin & 32 & 32 & $<4-32$ & $\leqslant 32(87 / 87)$ & $64(0 / 87)$ & $\geqslant 128(0 / 87)$ \\
\hline
\end{tabular}

*Sensitive, intermediate, and resistant are classified according to the NCCLS guidelines, 2002.

Osaka prefecture. The two cities adjoin one another and share the same cultural and economic background.

The proportion of isolates sensitive to ciprofloxacin, levofloxacin, and gatifloxacin was $10.3 \%, 27.6 \%$, and $21.8 \%$, respectively. The $\mathrm{MIC}_{90}$ of ciprofloxacin, levofloxacin, gatifloxacin, and moxifloxacin against the isolates was over $8 \mu \mathrm{g} / \mathrm{ml}$, over $8 \mu \mathrm{g} / \mathrm{ml}, 4 \mu \mathrm{g} / \mathrm{ml}$, and $2 \mu \mathrm{g} / \mathrm{ml}$, respectively (table 1). Median MICs of penicillin G were significantly higher for the isolates with resistance or reduced susceptibility to fluoroquinolones (ciprofloxacin, levofloxacin, and gatifloxacin) than fluoroquinolone susceptible isolates $(\mathrm{p}<0.00 \mathrm{l}, \mathrm{p}=0.0078$ and $\mathrm{p}=0.0079$, respectively $)$. Median MICs of tetracycline for isolates with resistance or reduced susceptibility to fluoroquinolones (ciprofloxacin, levofloxacin, and gatifloxacin) were not significantly different from those for isolates with fluoroquinolone susceptible isolates (table 2).

\section{Susceptibilities to penicillin and tetracycline}

The proportion of isolates sensitive to penicillin $G$ and tetracycline was $10.3 \%$ and $18.4 \%$, respectively (table 1 ). MIC $_{90}$ values of penicillin $\mathrm{G}$ and tetracycline against isolates were $2 \mu \mathrm{g} / \mathrm{ml}$ and $8 \mu \mathrm{g} / \mathrm{ml}$. One isolate each was categorised as PPNG and as TRNG. No isolate was classified as PPNGTRNG. Proportions of isolates with chromosomally mediated penicillin resistance (CMPR) and chromosomally mediated tetracycline resistance (CMTR) were $12.6 \%$ and $33.3 \%$. Seven isolates $(8.0 \%)$ were categorised to CMRNG.

\section{Susceptibilities to cephems and spectinomycin}

$\mathrm{MIC}_{90}$ values of cefixime, ceftriaxone, and spectinomycin against $N$ gonorrhoeae were $0.5 \mu \mathrm{g} / \mathrm{ml}, 0.063 \mu \mathrm{g} / \mathrm{ml}$, and 32 $\mu \mathrm{g} / \mathrm{ml}$, respectively. Proportions of isolates susceptible to cefixime, ceftriaxone, and spectinomycin were $90.8 \%, 100 \%$, and $100 \%$, respectively (table 1 ).

\section{DISCUSSION}

$N$ gonorrhoeae shows unique region specific susceptibility profiles. Moreover, a longitudinal survey in the same geographic area showed that susceptibilities changed over time. ${ }^{3-8}$

In our present study the proportion of isolates susceptible to penicillin $\mathrm{G}$ and tetracycline was $10.3 \%$ and $18.4 \%$, with a susceptibility profile fairly similar to those previously reported in Japan'; $90.8 \%$ of isolates were sensitive to cefixime, again in agreement with previous reports from other parts of Japan. ${ }^{6}{ }^{10}$ All isolates were found to show low MICs and susceptibility to ceftriaxone, which is used intravenously. This indicates that ceftriaxone can be a good choice of agent against $N$ gonorrhoeae in our area.

Proportions of $N$ gonorrhoeae resistant to fluoroquinolones differ from area to area worldwide. Prevalence of ciprofloxacin, levofloxacin, and gatifloxacin resistant $N$ gonorrhoeae in the present study proved to be $69.0 \%, 66.7 \%$, and $70.1 \%$, respectively. Since fluoroquinolones had been used as first line antimicrobial agents for $N$ gonorrhoeae infections in Japan, this heavy use created a selective advantage for microorganisms resistant to these drugs. ${ }^{3}$ We found these mutations in over $90 \%$ of isolates (Shigemura et al, unpublished data), they also can account readily for cross resistance among fluoroquinolones. For example, a new fluoroquinolone not yet commercially available in Japan, moxifloxacin, already showed relatively high MIC $_{90}$ values $(2 \mu \mathrm{g} / \mathrm{ml}$ ) (data not shown).

MICs of structurally unrelated penicillin, tetracycline, and cephems for the isolates with reduced susceptibility to ciprofloxacin have been reported to be higher than those for isolates susceptible to ciprofloxacin. Our study also demonstrated that MICs of penicillin G and cephems for isolates with reduced susceptibility to fluoroquinolones were almost 4-16 times higher than those for fluoroquinolone susceptible isolates. However, in the present study, MICs of tetracycline for isolates with reduced susceptibility to fluoroquinolones were the same as those for fluoroquinolone susceptible isolates. The reason for this discrepancy from previous reports is not evident from our present data, ${ }^{3}$ but is partly that tetracycline had not been used as often for gonococci treatment in Japan (table 2). Currently only one isolate each was categorised as PPNG or TRNG, and no isolate

Table $2 M^{2} C_{50}$ values of structurally unrelated antimicrobial agents for fluoroquinolone sensitive and fluoroquinolone less sensitive $\mathrm{N}$ gonorrhoeae

\begin{tabular}{|c|c|c|c|c|c|c|c|c|c|}
\hline \multirow[b]{2}{*}{ Antimicrobial agents } & \multicolumn{3}{|c|}{ Ciprofloxacin } & \multicolumn{3}{|c|}{ Levofloxacin } & \multicolumn{3}{|c|}{ Gatifloxacin } \\
\hline & Sensitive & Less sensitive* & $\mathrm{p}$ Value & Sensitive & Less sensitive* & p Value & Sensitive & Less sensitive ${ }^{*}$ & p Value \\
\hline Penicillin G & 0.031 & 0.5 & $<0.001$ & 0.375 & 0.5 & 0.0078 & 0.125 & 0.5 & 0.0079 \\
\hline Tetracycline & 0.25 & 1 & 0.508 & 1 & 1 & 0.2152 & 4 & 1 & 0.7423 \\
\hline Cefixime & 0.008 & 0.125 & $<0.001$ & 0.078 & 0.125 & 0.1599 & 0.016 & 0.125 & 0.0541 \\
\hline Ceffriaxone & 0.004 & 0.016 & 0.0006 & 0.01 & 0.016 & 0.0477 & 0.004 & 0.016 & 0.0384 \\
\hline Spectinomycin & 32 & 32 & 0.2022 & 32 & 32 & 0.4734 & 32 & 32 & 0.8326 \\
\hline
\end{tabular}

*Less sensitive includes the category of intermediate and resistant according to the NCCLS guidelines, 2002. 
was categorised as PPNG-TRNG; $12.6 \%, 33.3 \%$, and $8 \%$ of isolates were categorised as CMPR, CMTR, and CMRNG respectively. A previous report from another part of Japan showed less prevalence of CMPR, CMTR, and CMRNG, even though the first line treatment was the same in both areas. ${ }^{3}$

In Japan, currently recommended therapy against $N$ gonorrhoeae infection would be spectinomycin ( $2.0 \mathrm{~g}$ intramuscularly, given once) or a 3 day oral course of cefixime (200 mg twice a day). ${ }^{11}$ Yet in recent reports from Japan, susceptibility of $N$ gonorrhoeae to cefixime is decreasing. ${ }^{9}{ }^{10}$ Taking this, together with our results, spectinomycin and ceftriaxone are the current drugs of choice for treatment of $N$ gonorrhoeae infection in our region, meaning that we have no optimal oral regimen for treatment of $N$ gonorrhoeae. To establish a rational treatment strategy against $N$ gonorrhoeae infection, periodic surveys of MICs of $N$ gonorrhoeae isolated in particular areas are necessary, even when data have been reported from another part of the country. Moreover, specialists in treatment of STD should inform the other physicians who also treat patients with these infections of the current situation concerning resistance patterns and consequent adjustments in therapeutic strategy.

\section{Authors' affiliations}

K Shigemura, H Okada, T Shirakawa, K Tanaka, S Arakawa,

S Kinoshita, A Gotoh, S Kamidono, Division of Urology, Department of Organs Therapeutics, Faculty of Medicine, Kobe University Graduate School of Medicine, Kobe, Japan

\section{REFERENCES}

1 De Schryver A, Meheus A. Epidemiology of sexually transmitted diseases: the global picture. Bull World Health Organ 1990;68:639-54

2 Gorwitz RJ, Nakashima AK, Moran J, et al. Sentinel surveillance for antimicrobial resistance in Neisseria gonorrhoeae United States, 1988-1991. Morb Mortal Wkly Rep 1993;42(SS-3):29-39.

3 Tanaka M, Nakayama H, Haraoka M, et al. Antimicrobial resistance of Neisseria gonorrhoeae and high prevalence of ciprofloxacin-resistant isolates in Japan, 1993 to 1998. J Clin Microbiol 2000;38:521-5.

4 National Committee for Clinical Laboratory Standards. Performance standards for antimicrobial susceptibility testing. Informational supplement. M100-S12. Wayne, PA: National Committee for Clinical Laboratory Standards, 2002

5 Moodley P, Pillay C, Goga R, et al. Evolution in the trends of antimicrobial resistance in Neisseria gonorrhoeae isolated in Durban over a 5 year period: impact of the introduction of syndromic management. J Antimicrob Chemother 2001;48:853-9.

6 Muratani T, Akasaka S, Kobayashi T, et al. Outbreak of cefozopran (penicillin, oral cephems, and aztreonum) resistant Neisseria gonorrhoeae in Japan. Antimicrob Agents Chemother 2001;45:3603-6.

7 Dragsted D, Poulsen P, NØrgaard C, et al. Antimicrobial susceptibility of Neisseria gonorrhoeae strains isolated in Nuuk, Greenland during 19981999. Int J STD AIDS 2002;13:826-8.

8 Chalkley LJ, Rensburg MNJV, Matthee PC, et al. Plasmid analysis of Neisseria gonorrhoeae isolates and dissemination of tet $M$ genes in southern Africa 1993-1995. J Antimicrob Chemother 1997:40:817-822.

9 Deguchi T, Yasuda M, Yokoi S, et al. Treatment of uncomplicated gonococcal urethritis by double-dosing of $200 \mathrm{mg}$ cefixime at a 6-h interval. J Infect Chemother 2003;9:35-39.

10 Tanaka $\mathrm{M}$, Nakayama $\mathrm{H}$, Tunoe $\mathrm{H}$, et al. A remarkable reduction in the susceptibility of Neisseria gonorrhoeae isolates to cephems and the selection of antibiotic regimen for the single-dose treatment of gonococcal infection in Japan. J Infect Chemother 2002;8:81-6.

11 World Health Organization. Programme for sexually transmitted diseases, global programme on AIDS. Recommendations for the management of sexually transmitted diseases. WHO/GPA/TEM/94. Geneva: WHO, 1994. 